\title{
Estratégias de educação em saúde às gestantes e puérperas no enfrentamento à pandemia de COVID-19
}

\author{
Health education strategies for pregnant and puerperal women in coping with the COVID-19 \\ pandemic \\ Estrategias de educación para la salud para mujeres embarazadas y embarazadas para enfrentar la \\ pandemia del COVID-19
}

Recebido: 03/03/2021 | Revisado: 07/03/2021 | Aceito: 10/03/2021 | Publicado: 19/03/2021

\author{
Juliana Romano de Lima \\ ORCID: https://orcid.org/0000-0002-7404-6541 \\ Universidade Federal de Pernambuco, Brasil \\ E-mail: juliana_romano144@hotmail.com \\ Marcela Claudia de Paula Oliveira \\ ORCID: https://orcid.org/0000-0002-7762-0684 \\ Universidade Federal de Pernambuco, Brasil \\ E-mail: marcela-claudia2011@hotmail.com \\ Kelita Mirelle Oliveira Lima \\ ORCID: https://orcid.org/0000-0002-3783-2201 \\ Universidade Federal de Pernambuco, Brasil \\ E-mail: kelita_mirelle_sp@ hotmail.com \\ Aline Kelly de Moura \\ ORCID: https://orcid.org/0000-0002-0649-3323 \\ Universidade Federal de Pernambuco, Brasil \\ E-mail: alinek_moura@hotmail.com \\ José Jailson de Almeida Junior \\ ORCID: https://orcid.org/0000-0001-7448-0703 \\ Universidade Federal do Rio Grande do Norte, Brasil \\ E-mail: jailsonjrn@gmail.com \\ Vilma Costa de Macêdo \\ ORCID: https://orcid.org/0000-0003-3068-3175 \\ Universidade Federal de Pernambuco, Brasil \\ E-mail: vilacmacedo@hotmail.com
}

\begin{abstract}
Resumo
Objetivo: elencar as estratégias de educação em saúde voltadas às gestantes e puérperas no contexto da pandemia pelo COVID-19. Método: Revisão integrativa da literatura realizada através das Bases de dados da Medline, Cinahl e Lilacs utilizando o cruzamento dos descritores "Coronavirus infections", "Health Education" e "Pregnant Women" e seus análogos em Português e Espanhol. O processo de seleção dos artigos considerou as recomendações da Preferred Reporting Items for Systematic Reviews and Meta-Analyses. Foram encontrados 402 artigos, dentre os quais sete abordaram o tema proposto e foram selecionados para compor a amostra final deste estudo. Resultados: A pandemia pelo COVID-19 impôs medidas de contenção de novos casos acarretando o isolamento social. Estas mudanças contribuíram para que as mídias se tornassem locais privilegiados de trocas de informações. Instituições de ensino e saúde e profissionais de saúde utilizaram-se das redes sociais (Instagram®, Facebook® e WhatsApp®), rádios e "lives" para divulgarem e abordarem temáticas relacionadas à gestação, puerpério e o novo coronavírus. Os principais temas abordados foram os cuidados com a amamentação, prevenção, fluxo de atendimento às gestantes e puérperas com COVID-19 e preparação para o parto. Conclusão: As mídias e redes sociais permitiram às gestantes e puérperas o acesso às informações seguras baseadas nas recentes evidências científicas sobre o COVID-19, trazendo à tona um novo formato de estratégia educativa e contribuindo para a prevenção da doença emergente e promoção da saúde das mulheres.
\end{abstract}

Palavras-chave: Infecções por coronavírus; Educação em saúde; Gestantes; Promoção da saúde.

\section{Abstract}

Objective: to list health education strategies aimed at pregnant women and puerperal women in the context of the pandemic by COVID-19. Method: Integrative literature review carried out through the Medline, Cinahl and Lilacs databases using the crossing of the descriptors "Coronavirus infections", "Health Education" and "Pregnant Women" and their analogues in Portuguese and Spanish. The selection process of the articles considered the recommendations of the Preferred Reporting Items for Systematic Reviews and Meta-Analyses. 402 articles were found, among which 
seven addressed the proposed theme and were selected to compose the final sample of this study. Results: The COVID-19 pandemic imposed measures to contain new cases resulting in social isolation. These changes contributed to the media becoming privileged places for exchanging information. Educational and health institutions and health professionals used social networks (Instagram ${ }^{\circledR}$, Facebook ${ }^{\circledR}$ and WhatsApp ${ }^{\circledR}$ ), radio stations and "lives" to disseminate and address themes related to pregnancy, the puerperium and the new coronavirus. The main topics addressed were care with breastfeeding, prevention, flow of care for pregnant women and postpartum women with COVID-19 and preparation for delivery. Conclusion: The media and social networks allowed pregnant women and mothers to have access to safe information based on the recent scientific evidence on COVID-19, bringing to light a new format of educational strategy and contributing to the prevention of emerging disease and health promotion for women. women.

Keywords: Coronavirus infections; Health education; Pregnant women; Health promotion.

\section{Resumen}

Objetivo: enumerar estrategias de educación en salud dirigidas a gestantes y puérperas en el contexto de la pandemia por COVID-19. Método: Revisión integrativa de la literatura realizada através de las bases de datos Medline, Cinahl y Lilacs mediante el cruce de los descriptores "Infecciones por coronavirus", "Educación para la salud" y "Mujeres embarazadas" y sus análogos en portugués y español. El proceso de selección de los artículos consideró las recomendaciones de los Ítems Preferidos para Reportes para Revisiones Sistemáticas y Metaanálisis. Se encontraron 402 artículos, de los cuales siete abordaron la temática propuesta y fueron seleccionados para componer la muestra final de este estudio. Resultados: La pandemia COVID-19 impuso medidas para contener nuevos casos que resultaron en aislamiento social. Estos cambios contribuyeron a que los medios de comunicación se convirtieran en lugares privilegiados para el intercambio de información. Instituciones educativas, de salud y profesionales de la salud utilizaron las redes sociales (Instagram ${ }^{\circledR}$, Facebook ${ }^{\circledR}$ y WhatsApp ${ }^{\circledR}$ ) y estaciones de radio para difundir y abordar temas relacionados con el embarazo, el puerperio y el nuevo coronavirus. Los principales temas abordados fueron atención con lactancia materna, prevención, flujo de atención o COVID-19 y preparación para el parto. Conclusión: Los medios de comunicación y las redes sociales permitieron que las mujeres embarazadas y las madres tuvieran acceso a información segura basada en la evidencia científica reciente sobre COVID-19, sacando a la luz un nuevo formato de estrategia educativa y contribuyendo a la prevención de enfermedades emergentes y promoción de la salud para mujeres, mujeres.

Palabras clave: Infecciones por coronavírus; Educación para la salud; Mujeres embarazadas; Promoción de la salud.

\section{Introdução}

A COVID-19 é uma emergência de saúde pública de importância mundial, cuja apresentação clínica é variável de acordo com o perfil do paciente, apresentando sintomatologias leves à síndrome respiratória aguda grave (Brasil, 2020). O vírus foi identificado no final do ano de 2019, na Cidade de Wuhan, como causa de uma sequência de casos de pneumonia na China (Ortiz et al., 2020) sendo nomeado de SARS-CoV-2, do inglês Severe Acute Respiratory Syndrome Coronavirus 2, designado COVID-19 pela Organização Mundial de Saúde (OMS), a qual declarou o surto da doença como pandemia (Brasil, 2020, Ortiz et al., 2020).

As observações clínicas realizadas sobre a patologia apontam que a principal forma de contágio ocorre por meio do contato direto entre indivíduos, através das gotículas e aerossóis produzidos pelo espirro, tosse e até mesmo a fala da pessoa contaminada que entram em contato com a mucosa da boca, nariz ou olhos de pessoas saudáveis próximas, a menos de 1,80 metros de distância. O contágio por contato indireto é outra forma importante de transmissão, e acontece quando um indivíduo saudável encosta-se na superfície contaminada e conduz a mão aos olhos, nariz ou boca (Brasil, 2020, Jiang et al., 2020).

A elevada infectividade da COVID-19 funciona também como fator da rápida disseminação do vírus. O período médio de incubação da infecção é de cinco dias e a transmissibilidade em média, de 7 a 14 dias após o início dos sintomas (Jiang et al., 2020, Guo et al., 2020). Dentre as principais medidas de prevenção está a lavagem rigorosa das mãos com água e sabão, além da utilização de álcool gel ou líquido como solução sanitizante para desinfecção de mãos e superfícies (Jiang et al., 2020, Guo et al., 2020, Ramiro et al., 2020).

Segundo o Johns Hopkins Coronavírus Resource Center, até 26 de janeiro de 2021, foram notificados 99.839 .462 casos e 2.143.861 óbitos por COVID- 19 em todo o mundo. O Brasil ocupa o terceiro lugar no ranking com 8.871 .393 casos notificados, sendo o país com o segundo maior número de mortes pela doença com 217.664, ficando atrás apenas dos Estados 
Unidos da América (Johns Hopkins University, 2021).

Dados divulgados em junho de 2020 pela revista médica International Journal of Gynecology and Obstetrics revelaram que no mundo 160 grávidas ou puérperas morreram por complicações secundárias ao COVID-19 e destas 124 eram brasileiras (Takemoto et al., 2020). Neste contexto, o Brasil é responsável por 77\% das mortes de gestantes por coronavírus dentro do cenário mundial.

Estudos demonstram que as infecções causadas pelos vírus SARS-CoV, influenza H1N1 e MERS-CoV ocorridas nos anos 2002, 2009 e 2012, respectivamente, causaram nas gestantes sintomas como febre, tosse e dispnéia. O conhecimento adquirido a partir desses eventos sugere que mulheres grávidas e seus fetos são particularmente suscetíveis a desfechos ruins (Chattu \& Yaya, 2020, Rasmussen et al., 2008, 2012, Rodríguez-Blanco et al., 2020). Embora não haja dados de boa qualidade suficientes para tirar conclusões em relação à gravidade da doença ou complicações específicas de COVID-19 neste grupo, os estudos demonstram que a infecção está associada a uma maior taxa de nascimento prematuro, pré-eclâmpsia, cesárea e morte perinatal (Mascio et al., 2020, Zaigham \& Andersson, 2020, Zhu et al., 2020).

$\mathrm{O}$ isolamento social, condicionado pela pandemia, favoreceu a uma maior utilização das redes sociais por parte da população. A capilarização da informação possibilitou que as estratégias em saúde desenvolvidas através das redes alcançassem um maior engajamento e conscientização populacional quanto à forma de agir em tempos de COVID-19. Os serviços de saúde, por sua vez, reorganizaram suas rotinas de atendimento e as redes fortaleceram essa pactuação com a comunidade (Passadino et al., 2020, Melo et al., 2020, Rocha et al., 2020).

A educação em saúde no período gravídico-puerperal justifica sua importância por esta ser uma fase da vida da mulher em que as decisões tomadas são essenciais para os resultados maternos e neonatais (Shahidi et al., 2011, Kamau et al., 2019). Neste contexto, a internet vem se tornando nos últimos anos uma importante fonte de informações sobre saúde para esse grupo. A acessibilidade tornou-a uma mídia popular e têm trazido efeitos significativos na redução da taxa de mortalidade materna e infantil (Javanmardi et al., 2018, Ahmad et al., 2019). Essa ferramenta tem contribuído para a diminuição da exposição das mulheres às unidades de saúde e servem como aliadas no processo de conscientização e prevenção da COVID19 (Carvalho et al., 2020, Nery et al., 2020, Moreira et al., 2020).

Diante das complicações para a gestação impostas pela infecção pelo COVID-19 este estudo objetivou elencar as estratégias de educação em saúde voltadas às gestantes e puérperas no enfrentamento à pandemia de COVID-19.

\section{Metodologia}

O estudo utilizou como método de pesquisa a revisão integrativa da literatura. Percorreu as seguintes etapas: definição do problema, pesquisa nas bases de dados científicos, avaliação crítica das evidências encontradas e a discussão dos resultados obtidos. Tal prática encoraja a assistência à saúde pautada em conhecimento científico (Polit \& Beck, 2011).

Para o levantamento da questão norteadora, empregou-se a estratégia PICO (P - População: Gestantes e puérperas; I Intervenção: Estratégias de educação em saúde em tempos de COVID-19; C- Comparação: não se aplica; O - Desfecho: Análise das evidências científicas. Essa estratégia fundamentada na segmentação da hipótese, visa o levantamento de dados de forma sistematizada (Polit \& Beck, 2011). Desta forma, estipulou-se a seguinte questão norteadora da pesquisa: "Quais as estratégias de educação em saúde voltadas às gestantes e puérperas no enfrentamento à pandemia de COVID-19?"

O processo de seleção dos artigos considerou as recomendações PRISMA (Moher et al., 2015) (Preferred Reporting Items for Systematic Reviews and Meta-Analyses) (Figura 1). O levantamento dos dados ocorreu no mês de dezembro de 2020 nas Bases de Dados da Literatura Latino-Americana e do Caribe em Ciências da Saúde (LILACS), na Cumulative Index to Nursing and Allied Health Literature (CINAHL) e nos Bancos de Dados da Medical Literature Analysis and Retrieval System Online (MEDLINE/ PUBMED). Foram realizados cruzamentos dos descritores em inglês pelo Medical Subject Heading 
(MESH) "Coronavirus Infections", "Health Education" e "Pregnant Women"e em Português pelo Descritores em Ciências da Saúde (DeCS) "Infecções por Coronavirus", "Educação em Saúde" e "Gestantes".

Figura 1: Fluxograma das etapas referente à busca de evidências nas bases de dados, conforme o PRISMA, Recife, Pernambuco, Brasil, 2020.

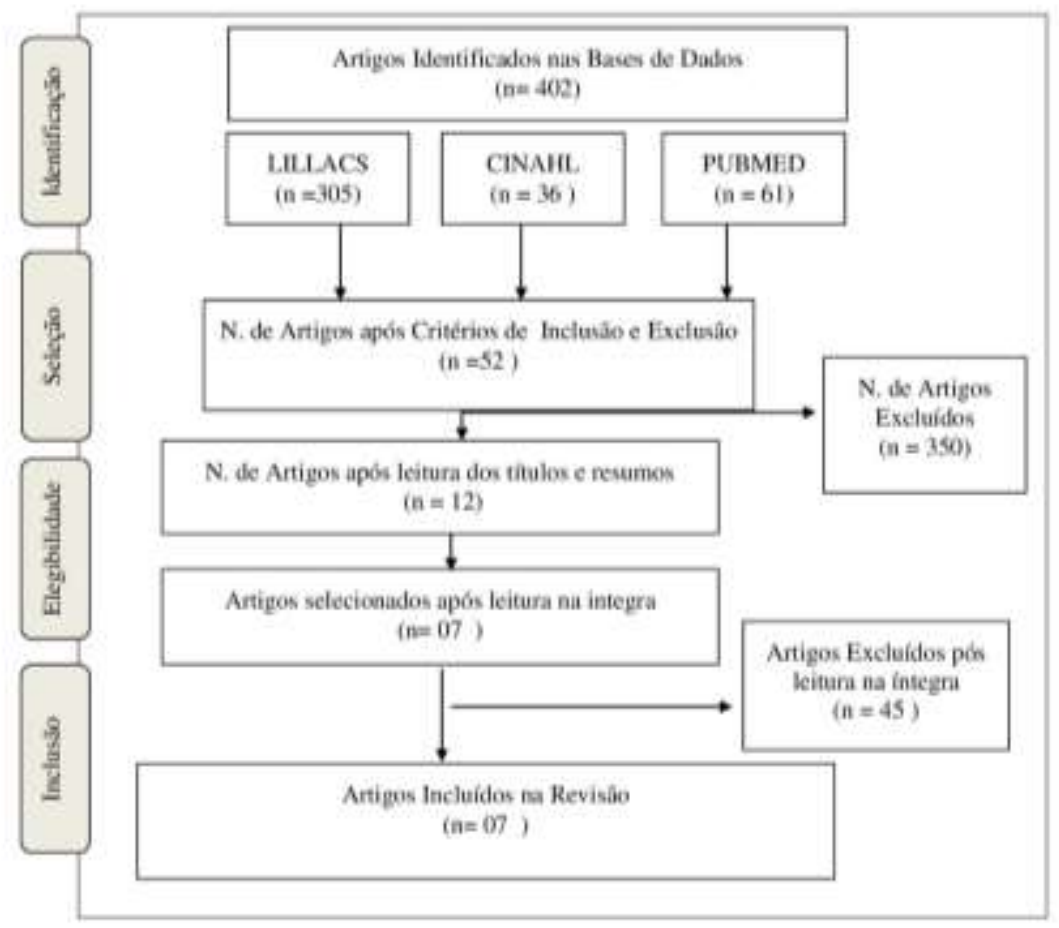

Fonte: Adaptado de The PRISMA Group (Moher et al., 2015).

Para definição das informações a serem extraídas dos artigos selecionados e categorização dos estudos utilizou-se a busca por pares, com o objetivo de fornecer uma maior credibilidade ao conteúdo da análise. Os descritores foram confrontados de forma pareada e depois em sequências combinadas com prioridade para o descritor "Health Education", a fim de padronizar os cruzamentos nas bases de dados.

Para seleção dos artigos, foi utilizado como critérios de inclusão: ser artigo original, publicado em português, inglês ou espanhol, disponível na íntegra. Não foi estabelecido recorte temporal uma vez que a identificação do vírus é recente (2019). Foram excluídos: as teses, as dissertações e as monografias, os editoriais, as revisões integrativas e as sistemáticas, além das repetições de publicação de estudos em mais de uma base de dados como também os artigos que não responderam à questão norteadora do estudo.

Os estudos que compuseram esta revisão foram classificados quanto à prática baseada em evidências, sendo caracterizados de forma hierárquica, utilizando o referencial americano da Agency for Healthcare Research and Quality (AHRQ) que considera o delineamento de pesquisa (Jeanne-Marie Guise, 2020).

Ressalta-se que a AHRQ classifica a qualidade das evidências em seis níveis: nível 1: metanálise de múltiplos estudos controlados; nível 2, estudo individual com delineamento experimental; nível 3, estudo com delineamento experimental como estudo sem randomização com grupo único pré e pós-teste, séries temporais ou caso controle; nível 4, estudo com delineamento não experimental como pesquisa descritiva correlacional e qualitativa ou estudo de caso; nível 5, relatórios de casos ou dado obtido de forma sistemática, de qualidade verificável ou dados de avaliação de programas; nível 6, opinião de autoridades respeitáveis baseada na competência clínica ou opinião de comitês de especialistas, incluindo interpretações de 
informações não baseadas em pesquisas (Jeanne-Marie Guise, 2020).

Após leitura e releitura dos artigos selecionados, foi realizada uma descrição e discussão dos resultados com a literatura pertinente, evidenciando o conhecimento produzido sobre o tema proposto.

\section{Resultados e Discussão}

Foram encontrados 402 artigos, dentre os quais sete abordaram o tema proposto e foram selecionados para compor a amostra final deste estudo. A Lilacs abrangeu $75 \%$ dos artigos $(n=6)$, seguida pela Pubmed (25\% n=1). A Cinahl não apresentou resultados para a busca realizada.

A especificidade do tema e o uso de artigos originais para composição da amostra funcionaram como uma prerrogativa para a quantidade de estudos selecionados. Porém a comunidade científica internacional e nacional esboça a necessidade de novas publicações sobre a temática (Guo et al., 2020, Melo et al., 2020, Nogueira et al., 2020, Ortiz et al., 2020, Passadino et al., 2020, Ramiro et al., 2020, Rocha et al., 2020, Zaigham \& Andersson, 2020).

No Quadro 1, as publicações foram organizadas em: autores e ano de publicação, base, nível de evidência (NE), título, objetivo e síntese. Um artigo selecionado para a amostra (25\%) foi publicado em inglês e seis (75\%) em português. Cinco estudos apresentavam relatos de experiências exitosas de educação em saúde voltados a gestante ou puérperas no contexto da COVID -19 (71\%) e dois abordagem metodológicas (29\%), que propunham criação de instrumentos voltados a este processo educativo em saúde.

Neste sentido, de acordo com o referencial da AHRQ, 29\% das publicações apresentaram nível de evidência 3 e $71 \%$ nível de evidência 4. Dado o pouco conhecimento disponível sobre o tema, os resultados demonstram boa qualidade metodológica e redução de viés.

Quadro 1: Distribuição dos estudos de acordo com título, autores, local, ano e periódico, Recife, Pernambuco, Brasil, 2020.

\begin{tabular}{|c|c|c|c|}
\hline $\begin{array}{l}\text { Autor / Ano / Base / Nível } \\
\text { de Evidência (NE) }\end{array}$ & Título & Objetivo & $\begin{array}{l}\text { Estratégia de } \\
\text { Educação em Saúde em } \\
\text { tempos de COVID-19 }\end{array}$ \\
\hline 1. Fernandes et al. & $\begin{array}{l}\text { Amamentação X COVID- } \\
\text { 19: Elaboração de um POP } \\
\text { destinado as etapas de } \\
\text { extração e armazenamento } \\
\text { do leite materno }\end{array}$ & $\begin{array}{l}\text { Elaborar um Procedimento } \\
\text { Operacional Padronizado } \\
\text { (POP) sobre formas seguras } \\
\text { de realizar a extração e } \\
\text { armazenamento do leite } \\
\text { materno em meio a } \\
\text { pandemia do novo } \\
\text { coronavírus }\end{array}$ & $\begin{array}{l}\text { Material elaborado com } \\
\text { orientações para cada etapa } \\
\text { da extração do leite } \\
\text { materno, incluindo a } \\
\text { paramentação e higiene da } \\
\text { mãe e dos equipamentos e } \\
\text { utensílios, oferta do leite de } \\
\text { forma segura para o bebê e } \\
\text { armazenamento }\end{array}$ \\
\hline $\begin{array}{l}\text { 2. Moreira, Nascimento \& } \\
\text { Marques. } \\
\qquad 2020 \\
\text { Lilacs } \\
\text { NE: } 4\end{array}$ & $\begin{array}{l}\text { Coronavírus e gestação no } \\
\text { espaço virtual: um processo } \\
\text { de ensino-aprendizagem } \\
\text { através da enfermagem }\end{array}$ & $\begin{array}{l}\text { Discutir sobre coronavírus } \\
\text { e gestação em espaço } \\
\text { virtual através do processo } \\
\text { de ensino-aprendizagem da } \\
\text { Enfermagem }\end{array}$ & $\begin{array}{l}\text { Transmissão virtual em } \\
\text { tempo real na página } \\
\text { oficial da liga "LAEGO" na } \\
\text { rede social Instagram }{ }^{\circledR} \text {, } \\
\text { sobre o tema "Coronavírus } \\
\text { e Gestação" }\end{array}$ \\
\hline
\end{tabular}


Research, Society and Development, v. 10, n. 3, e36610313501, 2021

(CC BY 4.0) | ISSN 2525-3409 | DOI: http://dx.doi.org/10.33448/rsd-v10i3.13501

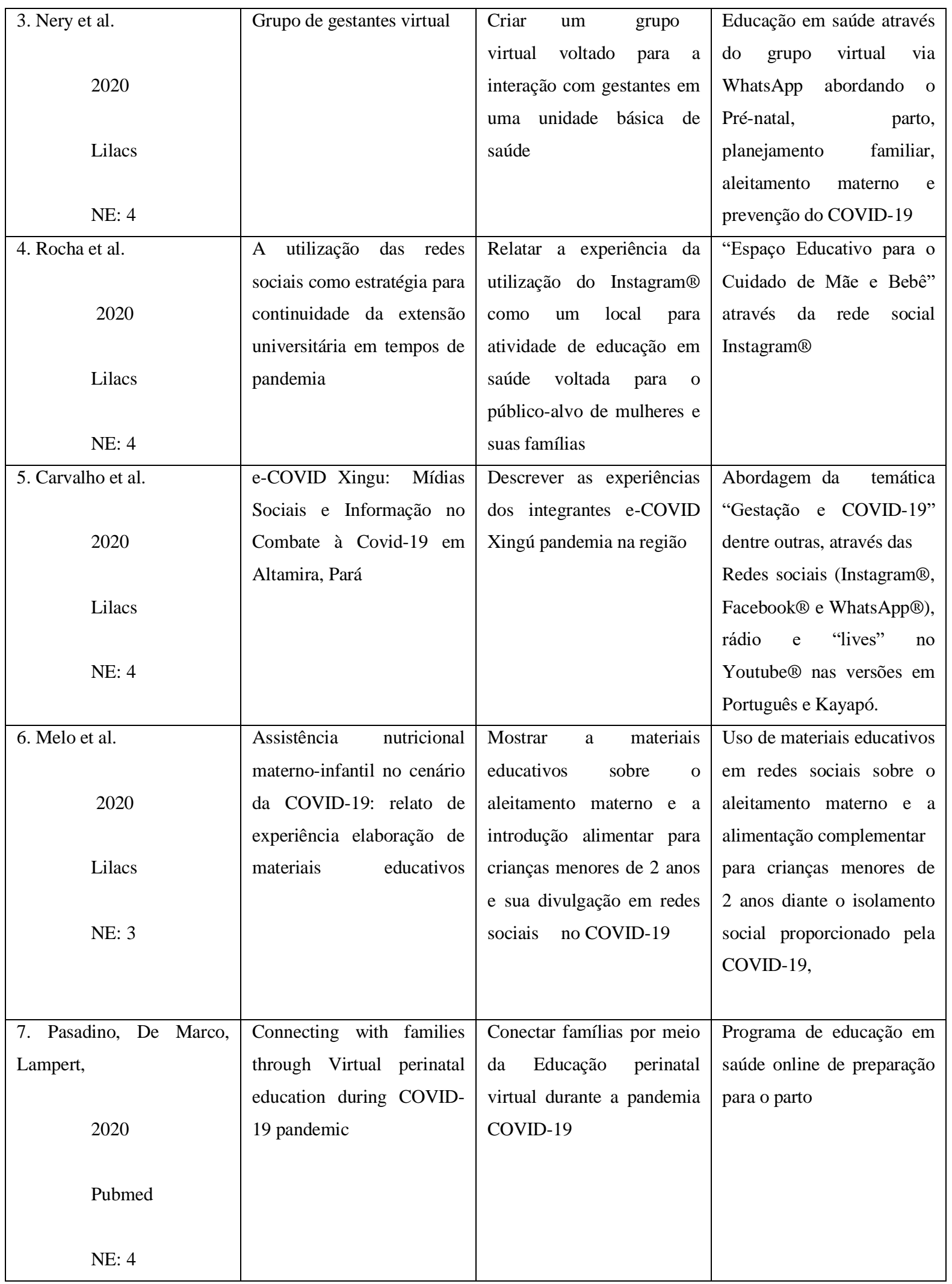

Fonte: Autores (2020).

Devido às questões relacionadas ao isolamento social imposto pelas medidas de contenção de novos casos pela COVID-19, as mídias sociais tornam-se locais privilegiados de trocas de informações durante a pandemia. As instituições de ensino e até mesmo alguns profissionais criaram páginas nas redes sociais e outras estratégias tais como fluxogramas para divulgar informações relacionadas à gestação, puerpério e o novo coronavírus (Carvalho et al., 2020, Melo et al., 2020, Nery et 
al., 2020, Passadino et al., 2020, Rocha et al., 2020).

Moreira, Nascimento e Marques (2020) se propuseram a analisar a questão do ensino-aprendizagem sobre gestação e o novo coronavírus nas ligas acadêmicas. Em decorrência da pandemia as pessoas conectaram-se mais na internet e plataformas como Instagram ${ }^{\circledR}$, Facebook ${ }^{\circledR}$ e WhatsApp ${ }^{\circledR}$ se transformaram em veículos de informação. Naquele estudo o Instagram ${ }^{\circledR}$ foi escolhido para vincular informações sobre transmissão, diagnóstico, prognóstico e tratamento da COVID-19 para gestantes. Visando maior alcance das informações a Liga organizou um cronograma de "Lives" (Transmissão ao vivo) (Moreira et al., 2020).

As transmissões dos vídeos em tempo real, propiciaram aos participantes a interação com o facilitador permitindo assim a discussão e construção de um novo cenário (pandemia) no tocante a gestação (Moreira et al., 2020). Foram abordadas temáticas sobre sintomatologia, cuidados, prevenção e fluxo de atendimento às gestantes com COVID-19 (Carvalho et al., 2020, Moreira et al., 2020, Nery et al., 2020, Rocha et al., 2020). Este tipo de estratégia para educação em saúde também foi elencada no estudo de Rocha et al (2020) que criou um canal no Instagram ${ }^{\circledR}$ denominado "Espaço Mãe-bebê", através do qual criou momentos de integração com intervalos semanais abordando a temática gestação, maternidade e COVID-19 (Rocha et al., 2020).

Para manter o vínculo com as gestantes cadastradas na unidade de saúde da família da Região da Tabajara (Uruguaiana-RS) a equipe utilizou a ferramenta WhatsApp ${ }^{\circledR}$ para formação de grupos a fim de contribuir para conscientização das usuárias com relação às ações e medidas que devem ser abordadas quando se trata do atual contexto sanitário mundial. Assuntos como importância do acompanhamento pré-natal e cuidados especiais frente à pandemia de COVID-19, tais como o planejamento individual para o momento do parto, apoio familiar e aleitamento materno além de medidas de prevenção ao contágio de COVID-19 foram lançados no grupo quinzenalmente possibilitando às gestantes um momento de troca de informação e aprendizagem (Nery et al., 2020).

Estas informações corroboram com os estudos desenvolvidos por Melo et al (2020) e Fernandes et al (2020) que ressaltaram a importância do aleitamento materno exclusivo até, no mínimo, o sexto mês de vida, utilizando, portanto, as ferramentas digitais e estratégias educativas a fim de atingir o maior quantitativo de gestantes e puérperas possíveis. Dentre as estratégias utilizadas, destaca-se a criação de um Protocolo Operacional Padrão (POP) sobre aleitamento materno, o qual abordou detalhadamente os cuidados necessários nas etapas de extração, armazenamento e processamento do leite materno de forma segura no contexto da pandemia pelo COVID-19 (Fernandes et al., 2020).

O estudo de Passadino et al. (2020) explana sobre a preparação para o parto no cenário pandêmico atual utilizando o formato virtual, demonstrando a importância das ações educativas em saúde através das redes sociais. O mesmo corrobora com os estudos brasileiros quando afirmam que a implementação dessa nova configuração de comunicação favoreceu um alcance maior do público alvo servindo como estratégia fundamental de prevenção à infecção por COVID -19 durante a gestação, hora do parto e amamentação (Carvalho et al., 2020, Moreira et al., 2020, Melo et al., 2020, Nery et al., 2020, Rocha et al., 2020).

Neste sentido, as redes sociais e outras demais estratégias educativas implementadas nesse contexto tais como a elaboração de materiais para divulgação, contribuíram para que às gestantes e puérperas conhecessem a importância e os benefícios da manutenção da amamentação, bem como do uso das máscaras de proteção e higienização das mãos para prevenção da doença pelo COVID-19.

\section{Conclusão}

A pandemia do COVID-19 impôs medidas para mudanças no comportamento humano tais como a lavagem das mãos, uso de máscaras faciais e luvas de proteção e distanciamento social. As mídias sociais através da internet contribuíram para as ações de promoção da saúde para os governos alcançarem as mudanças necessárias. A funcionalidade desta se mostrou uma 
potente ferramenta de educação em saúde, permitindo e facilitando a integração entre as gestantes, puérperas e os facilitadores da informação.

Universalizar o acesso à internet e investir em políticas públicas de promoção da saúde são necessárias para a continuidade da oferta do cuidado voltado às gestantes, puérperas e demais usuários do sistema público de saúde, a fim de garantir a continuidade do enfrentamento dos problemas de saúde pública vigentes. Consideramos o desenvolvimento de novos estudos a fim de elucidar as dificuldades impostas no desenvolvimento das ações educativas no contexto de pandemia pelo COVID-19.

\section{Referências}

Ahmad, R., Zhu, N. J., Lebcir, R. M., \& Atun, R. (2019). How the health-seeking behaviour of pregnant women affects neonatal outcomes: findings of system dynamics modelling in Pakistan. BMJ global health, 4(2), e001242.

Brasil. Ministério da Saúde. Departamento de ações programáticas estratégicas. Atenção às Gestantes no Contexto da Infecção COVID 19 causada pelo Novo Coronavírus (SARSCoV-2). 2020.

Carvalho, L. M., Nascimento, F. A. A., Granato, R. R., Damasceno, O. C., Texeira, F. B., Sato, D. A. (2020). e-COVID Xingu: Mídias Sociais e Informação no Combate à Covid-19 em Altamira, Pará. Revista Brasileira de Educação Médica, 44 (1), e0142.

Chattu, V. K., \& Yaya, S. (2020). Emerging infectious diseases and outbreaks: implications for women's reproductive health and rights in resource-poor settings. Colombia Médica, 51(2), e-4271.

Di Mascio, D., Khalil, A., Saccone, G., Rizzo, G., Buca, D., Liberati, M., \& D’Antonio, F. (2020). Outcome of coronavirus spectrum infections (SARS, MERS, COVID-19) during pregnancy: a systematic review and meta-analysis. American journal of obstetrics \& gynecology MFM, 2 (2), 100107.

Fernandes, E., Sousa, J., Silva, R., Serquiz, A. (2020). Elaboração de um POP destinado às etapas de extração e armazenamento do leite materno. Revista Extensão \& Sociedade: Especial Covid-19, 12(1):30-45.

Guo, Y.R., Cao, Q. D., Hong, Z. S., Tan, Y. Y., Chen, S. D., Jin, H. J., \& Yan, Y. (2020). The origin, transmission and clinical therapies on coronavirus disease 2019 (COVID-19) outbreak-an update on the status. Military Medical Research, 7(1), 1-10.

Javanmardi, M., Noroozi, M., Mostafavi, F., \& Ashrafi-Rizi, H. (2018). Internet usage among pregnant women for seeking health information: a review article. Iranian journal of nursing and midwifery research, 23(2), 79.

Jeanne-Marie Guise, M. D. (2020). AHRQ Series on Improving Translation of Evidence: Progress and Promise in Supporting Learning Health Systems. The Joint Commission Journal on Quality and Patient Safety, 46:51-52.

Jiang, F., Deng, L., Zhang, L., Cai, Y., Cheung, C.W., \& Xia, Z. (2020). Review of the clinical characteristics of coronaviru s disease 2019 (COVID-19). Journal of general internal medicine, 35(5), 1545-1549.

Johns Hopkins University. (2021). COVID-19 Map. Johns Hopkins Coronavirus Resource Center.

Kamau, M., Mirie, W., Kimani, S., \& Mugoya, I. (2019). Effect of community based health education on knowledge and attitude towards iron and folic acid supplementation among pregnant women in Kiambu County, Kenya: A quasi experimental study. PLoS One, 14(11), e0224361.

Melo, A. T. A., Carneiros, B. R., Silva, E. A. F., Rebouças, A. S., Dametto, J. F. S. (2020). Assistência nutricional maternoinfantil no cenário da covid-19: relato de experiência na elaboração de materiais educativos. Revista Extensão \& Sociedade: Especial Covid-19, 12(1):213-222.

Moher, D., Liberati, A., Tetzlaff, J., Altman, D. G. (2015). The PRISMA Group. Preferred Reporting Items for Systematic Reviews and Meta-Analyses: The PRISMA Statement. Epidemiol. Serv. Saúde, 24(2): 335-342.

Moreira, M. A., Nascimento, O. S., Marques, P. F. (2020). Coronavírus e gestação no espaço virtual: um processo de en sino-aprendizagem através da enfermagem. Revista Extensão \& Sociedade: Especial Covid-19, 12(1), $81-89$.

Nery, J., Alves, B. G. C., Ferreti, E., Souza, J. M. M., Raguzzoni, V. P., Rezer, J. F. P. (2020). Grupo de gestantes virtual. Anais do 12 SIEPE - Salão Internacional de Ensino, Pesquisa e Extensão da UNIPAMPA, 12(3): 11-13.

Nogueira, C. M. C. S., Alcantara, J. R., Costa, H. M. S. G. S., Morais, F. R. R., Bezerra, K. P., Fialho, A.V.M. (2020). Análise nacional do perfil das gestantes acometidas pela COVID-19. Braz. J. Hea. Rev, 3(5): 14267-14278.

Ortíz, E. I., Herrera, E., \& De la Torre, A. (2020). Infección por coronavirus (COVID-19) en el embarazo. Colomb Med (Cali), 51(2).

Osanan, G. C., Bonomi, I. B. A., Bacha, C. A., Laranjeiras, C. L. S., Ramos Filho, F. L., \& Aguiar, A. L. P. (2020). Coronavirus na gravidez: considerações e recomendações sogimig. SOGIMIG.

Passadino, F., De Marco, K., \& Lampert, E. (2020). Connecting with families through Virtual perinatal education during the covid-19 pandemic. MCN Am J Matern Child Nurse, 45(6):364-370.

Polit, D. F., \& Beck, C. T. (2011). Fundamentos da pesquisa em enfermagem: avaliação de evidências para a prática de enfermagem. ArtMed Editora. 
Research, Society and Development, v. 10, n. 3, e36610313501, 2021

(CC BY 4.0) | ISSN 2525-3409 | DOI: http://dx.doi.org/10.33448/rsd-v10i3.13501

Ramiro, N. C. M. P., de Souza Pereira, M., de Souza, R. S., \& Aver, L. A. (2020). Repercussões fetais e possíveis complicações da COVID-19 durante a gestação. Saúde Coletiva (Barueri), 10(54), 2679-2690.

Rasmussen, S. A., Jamieson, D. J., \& Bresee, J. S. (2008). Pandemic influenza and pregnant women. Emerging infectious diseases, $14(1)$, 95.

Rasmussen, S. A., Jamieson, D. J., \& Uyeki, T. M. (2012). Effects of influenza on pregnant women and infants. American journal of obstetrics and gynecology, 207(3), S3-S8.

Rocha, C. R., Moreira, A. P. A., da Silva, L. R., dos Santos, I. M. M., Barbosa, M. N., Bittencourt, G., \& Feitosa, I. B. (2020). A utilização das redes sociais como estratégia para continuidade da extensão universitária em tempos de pandemia. RAÍZES E RUMOS, 8(1), 261-269.

Rodríguez-Blanco, N., Vegara-Lopez, I., Aleo-Giner, L., \& Tuells, J. (2020). Revisión exploratoria sobre series de casos de coronavirus (SARS-CoV, MERSCoV y SARS-CoV-2) y sus resultados obstétricos y neonatales. Revista Española de Quimioterapia, 33(5), 313.

Santos, M. A. R. C., \& Galvão, M. G. A. (2014). A elaboração da pergunta adequada de pesquisa. Resid Pediatr, 4(2):53-56.

Shahidi, S., Aghdak, P., Farajzadegan, Z., Izadi, M., Mohammadi, M., \& Fard, M. N. (2011). Reviewing the effectiveness of pre-pregnancy counseling protocol on pregnancy and labor indices. Iranian journal of nursing and midwifery research, 16(4), 265.

Takemoto, M. L., Menezes, M. D. O., Andreucci, C. B., Nakamura-Pereira, M., Amorim, M. M., Katz, L., \& Knobel, R. (2020). The tragedy of COVID-19 in Brazil: 124 maternal deaths and counting. International Journal of Gynecology \& Obstetrics, 151(1), 154-156.

Zaigham, M., \& Andersson, O. (2020). Maternal and perinatal outcomes with COVID-19: a systematic review of 108 pregnancies. Acta obstetricia et gynecologica Scandinavica, 99(7), 823-829.

Zhu, H., Wang, L., Fang, C., Peng, S., Zhang, L., Chang, G., \& Zhou, W. (2020). Clinical analysis of 10 neonates born to mothers with 2019-nCoV pneumonia. Translational pediatrics, $9(1), 51$. 